\title{
June Updates: People and Places
}

\begin{abstract}
Zebrafish researcher recognized University of Oregon scientist Judith Eisen has recently been selected for membership in the American Academy of Arts and Sciences. Eisen was an early adopter of the zebrafish as a model animal to study the nervous system and is a principal investigator and founding member of the Zebrafish Model Organism Database (ZFIN), a resource dedicated to the fish and its use in research. The Academy, founded in Cambridge, Massachusetts in 1780 by John Adams, John Hancock, and James Bowdoin, honors "exceptional scholars, leaders, artists, and innovators and engages them in sharing knowledge and addressing challenges facing the world." Eisen is one of six fellows chosen in the area of the neurosciences, cognitive sciences, and behavioral biology. There were a total 213 Fellows elected for 2018; an induction ceremony will be held in the fall in Cambridge.
\end{abstract}

\section{A fellowship for systematic reviews}

Scottish stroke scientist and systemic review proponent Malcolm Macleod has been awarded the inaugural Maria Sibylla Merian Fellowship from the German Federal Institute for Risk Assessment (BfR). Macleod is Professor of Neurology and Translational Neurosciences at the University of Edinburgh and the coordinator of CAMARADES (Collaborative Approach to Meta-analysis and Review of Animal Data from Experimental Studies). The Fellowship recognizes Macleod's efforts to improve the reproducibility and translational value of animal research through systemic reviews and meta-analyses.

\section{Swine ok'd by the FDA \\ Exemplar Genetics, a company based in Sioux Center, Iowa that specializes in the development of genetically engineered minipigs for biomedical research, has received approval from the FDA for the commercial use of its ExeGen ATM MiniSwine model. These animals model ataxia telangiectasia, a rare and inherited neurodegenerative disease that disrupts motor function in human patients and currently has few treatment options. FDA approval expands access to the minipig to researchers and drug developers. \\ The model is Exemplar's second to be approved for commercial use by the FDA; the first, in 2016, was the ExeGen ${ }^{\circ}$ LDLR (low-density lipoprotein receptor) MiniSwine, which is available for cardiovascular research.}

\section{Oxytocin research continues at Emory The National Institute of Mental Health will continue funding to Emory University's Silvio O. Conte Center for Oxytocin and Social Cognition with a five-year, $\$ 12.7$ million dollar grant. The Conte Center was established in 2013 to study oxytocin's function in the brain and how it contributes}

\section{Careers update}

Veterinarian Karen Rogers will join West Lafayette, Indiana-based preclinical contract research organization Bioanalytical Systems, Inc (BASi) as an attending veterinarian for the company's preclinical pharmacokinetics/pharmacodynamics services. Philip Downing, vice president of preclinical services at BASi, commented in a press release, "Karen Rogers is an experienced lab animal veterinarian with a proven history of providing exceptional clinical care to a variety of laboratory animals.... We are confident that Karen's consultation and leadership skills will further support this commitment while strengthening our IACUC."

Rogers received her veterinary degree from the Ohio State University College of Veterinary Medicine. She completed a laboratory animal medicine residency program at the University of Michigan Medical School and is a Diplomate of the American College of Laboratory Animal Medicine. She is also an attending veterinarian and the Director of Laboratory Animal Resources at Indiana University.

to neural communication and social functioning through the use of rodent and nonhuman primate models. It is led by Emory scientist Larry Young and includes collaborators at the Yerkes National Primate Research Center, Emory, and the University of Arizona.

\section{New Alzheimer's chair at JAX}

Catherine Kaczorowski, a neuroscientist at the Jackson Laboratory in Bar Harbor, ME, will be the first chairholder of the Evnin Family Endowed Chair in Alzheimer's Research. The Evnin Family donated \$1.5 million to establish the position, which the Jackson Laboratory's board of trustees has matched.

Rather than focus on risk factors and susceptibility to Alzheimer's, Kaczorowski is interested in resilience; her lab uses mouse models to study potentially protective factors against the disease. "Sometimes in science we need to flip the question on its head: stop focusing on risk and start focusing on resilience. My hope is that the identification of these resilience factors will unmask new therapeutic strategies that no one has ever thought of before," she commented in a press release.

"Dr. Kaczorowski is approaching Alzheimer's from a novel perspective," said Anthony Evnin (also a Jackson Laboratory trustee). "We're thrilled to support the innovative Alzheimer's research under way in her laboratory."

\section{Election time}

The American Association for Laboratory Animal Science (AALAS) is getting ready to hold elections for its Board of Trustees. Several candidates are running for the following positions: Vice President-Elect; Secretary/Treasurer; At-Large Trustee seats 1 and 2; District 1 Alternative Trustee; District 3 Trustee; and District 5 Alternative Trustee.

Biographies for the candidates can be found on the AALAS website, and voting will begin on June 1, 2018.

Published online: 23 May 2018 https://doi.org/10.1038/s41684-018-0074-z 